\title{
A Comprehensive Review of Image Segmentation Techniques
}

\author{
Salwa Khalid Abdulateef*, Mohanad Dawood Salman \\ Department of Computer Science, Computer Science and Mathematics College, Tikrit University, Iraq.
}

\author{
Correspondence \\ * Salwa Khalid Abdulateef \\ Computer Science and Mathematics College, \\ Tikrit University, Iraq \\ Email: khalid.salwa@tu.edu.iq
}

\begin{abstract}
Image segmentation is a wide research topic; a huge amount of research has been performed in this context. Image segmentation is a crucial procedure for most object detection, image recognition, feature extraction, and classification tasks depend on the quality of the segmentation process. Image segmentation is the dividing of a specific image into a numeral of homogeneous segments; therefore, the representation of an image into simple and easy forms increases the effectiveness of pattern recognition. The effectiveness of approaches varies according to the conditions of objects arrangement, lighting, shadow and other factors. However, there is no generic approach for successfully segmenting all images, where some approaches have been proven to be more effective than others. The major goal of this study is to provide summarize of the disadvantages and the advantages of each of the reviewed approaches of image segmentation.
\end{abstract}

KEYWORDS: Clustering, edge detection, graph-cut, image segmentation, region, thresholding.

\section{INTRODUCTION}

An image is a visible representation of something and carries a lot of beneficial information. Analyzing the image and obtaining information from it in a way that does not influence the image's other features in order to get some tasks done is one of the important applications of digital image technology [1]. In various sectors and real-world applications such as military, medical, astronomy, etc. pattern recognition, image analysis, and image disciplines are the most important subjects in computer science and computer engineering [2].

Nowadays, ingenious technologies are developing in the domains of image processing, particularly in the image segmentation field. Image segmentation is an important and difficult process [3]. The image segmentation process is the most significant phase of image analysis. The technique of splitting an image into homogenous parts based on particular criteria and, ideally, corresponding to actual things in the scene is known as image segmentation [4]. In reality, image segmentation is utilized in the next phases of a standard recognition system to present simpler and easier data such as color or texture [5]. From a multi-media perspective, image segmentation can be applied to a single image or a series of images that form a video [6, 7]. In general, the basic goal of segmentation is for reducing data for an easier analysis process, where image segmentation is described as the division of a digital image into its continuous, unconnected, and nonempty subsets to facilitate attribute extraction [8]. Image segmentation is still an open and exciting research topic in the realm of image processing. The development of a universal technique for image segmentation remains a difficult task for academics and developers [9]. Basic prerequisites for good image segmentation include:

- Each pixel in the region belongs to an image.

- If any two pixels in a specified region may be connected by a line that does not exit the region, the region is linked

- Each region is homogeneous in term of a selected characteristic. The characteristic could be syntactic (intensity, color and texture) or semantically based.

- It is impossible to combine neighboring regions into a single homogeneous region.

- There is no overlap between regions [10]

The main purpose of the segmentation is to simplify an image, i.e. to represent it in a way that is expressive and easy in order to guarantee only the objects of interest are analyzed during the object analysis phase [11]. Nevertheless, a wrong segmentation will induce degradation of the classification process and object measurement [12].

The article's remaining sections are organized as follows. In section 2, image segmentation techniques are described. Next, section 3 addressed image segmentation applications. After that, the outcomes and discussions are provided in section 4 . Finally, in section 5 the conclusion and future works are presented.

This is an open access article under the terms of the Creative Commons Attribution License, which permits use, distribution and reproduction in any medium, provided the original work is properly cited.

(c) 2021 The Authors. Published by Iraqi Journal for Electrical and Electronic Engineering by College of Engineering, University of Basrah. 


\section{IMAGE SEgmentation TEChNIQUES}

The majority of image segmentation algorithms may be divided into three techniques: boundary-based segmentation, region-based segmentation, and hybrid-based segmentation [13].

The first technique relies on discontinuity to split an image by recognizing lines, edges, and isolated points based on abrupt changes in local attributes. The boundaries of the regions are subsequently inferred. To create the segmentation result, the second technique uses the homogeneity of spatially dense information such as texture, intensity, color. The third technique is combining between boundary-based segmentation and region-based segmentation as in Fig. 1. Besides, Table 1 present summarizes these techniques.

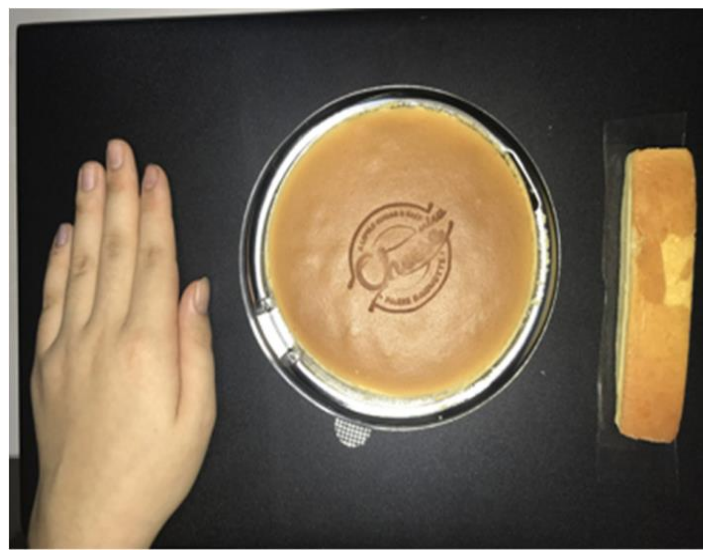

The existing segmentation methods usually separate objects from background only, pre-knowledge about data, User-specified input parameters are required, which might lead to under-segmentation or over-segmentation, control on environment [14-17]. Therefore, improvement of the current techniques, thus enable better classification of images. Despite the huge amount of the literature in the topic of image segmentation and the variety of methods that were used, Segmentation can be accomplished by different categories such as thresholding [18,19], edge-based segmentation [20, 21], region based segmentation [22- 25] and energy based segmentation [26-28]. Therefore, the following sub sections begin by reviewing thresholding based segmentation followed by the other three categories.

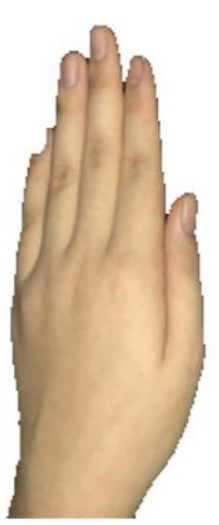

Fig. 1: Example of hybrid image segmentation [29]

TABLE 1. COMPARISON BETWEEN IMAGE SEGMENTATION TECHNIQUES

\begin{tabular}{|c|l|l|l|}
\hline Technique & \multicolumn{1}{|c|}{ Description } & \multicolumn{1}{|c|}{ Advantages } & \multicolumn{1}{c|}{ Disadvantages } \\
\hline $\begin{array}{c}\text { Boundary - based } \\
\text { segment }\end{array}$ & $\begin{array}{l}\text { Based on the } \\
\text { discontinuity } \\
\text { detection. }\end{array}$ & $\begin{array}{l}\text { 1- Suitable for simple image. } \\
\text { 2-It is very useful for images with a } \\
\text { large contrast between their } \\
\text { objects. } \\
\text { 3- Low computation intensive is } \\
\text { making the methods more ideal for } \\
\text { use. }\end{array}$ & $\begin{array}{l}\text { 1-It doesn't function well with } \\
\text { images that have a lot of edges or } \\
\text { not clear. } \\
\text { 2-Producing a closed curve or } \\
\text { boundary is not. } \\
\text { 3-It is not suitable for images have } \\
\text { very noisy. }\end{array}$ \\
\hline $\begin{array}{c}\text { Region-based } \\
\text { segment }\end{array}$ & $\begin{array}{l}\text { Similarity in term } \\
\text { of a set of } \\
\text { predefined criteria. }\end{array}$ & $\begin{array}{l}\text { 1- More impervious to noise. } \\
\text { 2-When defining similarity criteria is } \\
\text { straightforward, this method is } \\
\text { useful. }\end{array}$ & $\begin{array}{l}\text { Expensive according to memory and } \\
\text { time. }\end{array}$ \\
\hline $\begin{array}{c}\text { Hybrid - based } \\
\text { segment }\end{array}$ & $\begin{array}{l}\text { Based on } \\
\text { combining region } \\
\text { and edge. }\end{array}$ & $\begin{array}{l}\text { Depending on the combination of } \\
\text { techniques. }\end{array}$ & $\begin{array}{l}\text { Depending on the combination of } \\
\text { techniques. }\end{array}$ \\
\hline
\end{tabular}




\section{A. THRESHODING-BASED SEGMENT}

This category of approaches depends on the changes in the grey scale values of the image to divide it into subregions. It can also be used for extracting foreground objects from the background by selected threshold value .A gray level image can be converted to a binary image. All of the relevant information regarding the position and shape of the items of interest should be contained in the binary image. The advantage of obtaining a binary image is that it reduces data complexity and simplifies the recognition operation. Significant peaks and dips in the image are difficult to identification. However, the limitations are it does not take into account the spatial detail so the segmented regions may not be contiguous, it is sensitive to noise and difficult to set threshold [30]. Another disadvantage is the concern about the computational complexity, which increases proportionally when the size of the image increases [19]. Fig.2. shows example of threshold-based segment.

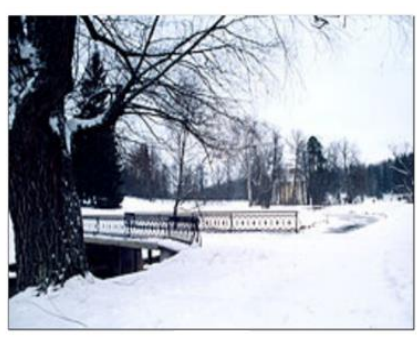

(a)

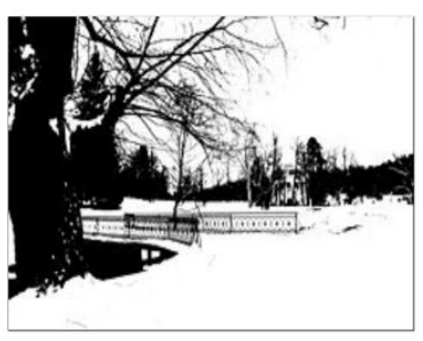

(b)
Fig. 2: Threshold-based segment (a) original image, (b) the resulting image after segmentation [31]

\section{B. EDGE-BASED-SEGMENT}

Segmentation in this class is performed by identifying the edges in the image on the gradient of the image with the aim to produce the boundaries of the objects. In this method, objects are identified through considering edges as criteria. It is generally used to identify these objects which include first order derivative/gradient operator, second derivative operator and optimal edge detector.

1. Gradient Operator which involves first order derivatives responds whenever there is discontinuity in intensity level. It has a leading edge that is positive and a following edge that is negative, such as Prewitt, Roberts and Sobel operators to detect edges by finding the magnitude in its first derivative $[21,32]$.

2. Second Derivative Operator when the darker side is positive, and the lighter side is negative. It is particularly sensitive to the noise in an image. But it is very useful for extracting some secondary information, such as Laplacian operator, and Difference of Gaussian (DoG) detect edges by searching for zero-crossings [33].

3. Optimal Edge Detector as canny edge detector can produce continuous edges, single-pixel thick, noise-resistant. It can also detect strong and weak edges [21, 34].

The edge-based segment is weak due to several factors. The first factor is because the technique fails in producing the boundary of the objects. The reason is that it allows for missing and disjointed edges in separating the area of interest and background. For most of the cases, the edges that were produced by this technique were disjoint. The second factor is its noise sensitivity. As a result, for noisy images, the edge frequently failed to achieve correct segmentation. The third factor is its sensitivity to expanding of edges between important regions. These difficulties occur in images with high spatial resolution and complex geometric shapes. Consequently, the edges between regions are hardly defined, thus producing over or under segmentation. An example of edge-based segment is shown in Fig. 3.

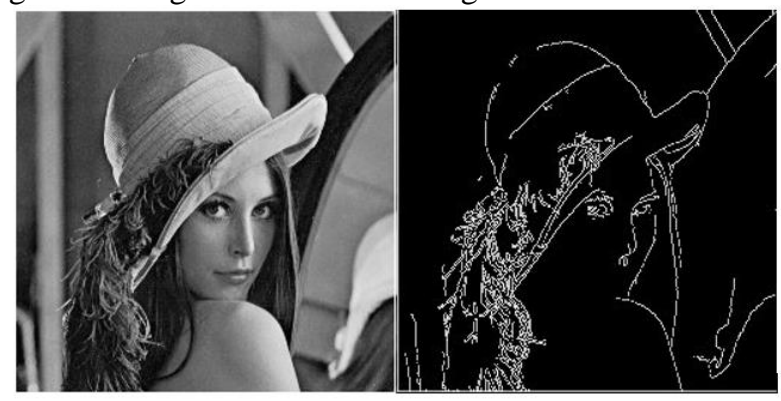

(a)

(b)

Fig. 3: Edge-based segment (a) original image, (b) the resulting image after segmentation [34]

\section{REGION-BASED SEGMENT}

This category is combination of region growing, splitting and merging and clustering.

1. Region Growing is a method in which a pre-defined criteria is used to group the entire image into sub-regions. However, this method suffers from the following drawbacks: sensitivity to noise, holes or discontinuity in the extracted region and high computational complexity $[35,36]$.

2. Region Splitting and Merging partitioned image into set of separated sub-regions. Next, a merging and/or splitting operation is performed on each sub-region according to a given criteria for segmentation. This method suffers from complexity and high-compositionality [37].

These methods are also unpopular due to several limitations. First is computationally more complex than other techniques, thus increasing the computational time and memory. Second limitation is due to its difficulties in extracting geometric information and therefore it is not suitable for images with shadow or shading. Due to the limitation of the region-based segmentation, the segmentation process requires paying attention to those limitations in order to deliver more precise and reliable methods.

3. Clustering is a arrange data into groups named clusters; each cluster contains data more similar to each other than others [38]. Clustering is applied in numerous fields, including medical [39], geographic [40, 41], and agriculture [42]. Clusters are created based on diversity of the properties such as size, color and texture [43]. Two types of clusters are considered: hierarchical which data is organized into tree data structure, the root represents the entire database, and the internal nodes stand for the cluster $[44,45]$. The second type 
is partition where pixels are divided into $\mathrm{k}$ cluster. K-means algorithm is used for classifying image pixels into $\mathrm{k}$ number of clusters where $\mathrm{k}$ is a whole positive number. The algorithm of K-means proposed by MacQueen [46] which has been used for segmenting images in several areas such as calories estimation [47, 48], and medical [39, 49]. This classification is performed based on some similarity features such as intensity of pixels, color and distance [43]. It is important to notice that the advantage of using clustering is that the non-need of prior knowledge about distribution of the data. Efficiency, straightforwardness, easy implementation and ability to cluster huge data points very quickly have attracted researchers to do k-means for image segmentation. This algorithm suffers from several drawbacks as lacking right criteria to define the number $\mathrm{k}$ that is required; results are not the same when execution is repeated and high dependency on the initial conditions. However, the k-mean technique has several limitations. Firstly, is sensitive to noise. Secondly, it has limited choice numbers of the cluster. Thirdly, various initial centroids produce different outcome and computationally more complex, thus increasing the computational time. Fig.4. illustrates a region-based segment.

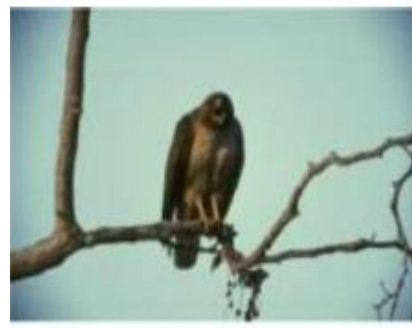

(a)

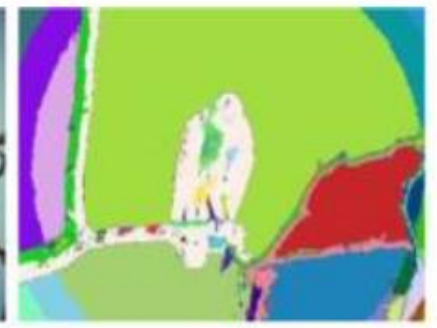

(b)
Fig. 4: Region-based segment (a) original image, (b) the resulting image after segmentation [50]

\section{ENERGY-BASED SEGMENT}

This category aims at performing an optimization of an objective energy function. The minimum solution is corresponding to the segmentation results. Active contour and graph-based method are the most common methods in this category.

1. Active Contour: active contour models have been broadly connected to image segmentation [51]. In the last decade, it has been widely used in a variety of applications including image segmentation and motion tracking. Essentially, active contour aims at deforming an initial curve to the boundary of an object subject to some constraints from the image. Snakes and level sets are the two basic models examined in active contour from an implementation standpoint. Snakes move predetermined snake spots according to an energy minimization strategy. In level set, contour is moved explicitly based on a particular level of function.

Typically, active contour models are used due to have many attractive favorable circumstances compared to traditional image segmentation methods, for example, region growing, thresholding and edge detection and. In the first place, active contour models can be effectively figured under a principled energy minimization framework and permit consolidation of different earlier knowledge. Second, they can give smooth and closed contours as a segmentation output, which are fundamental and may be quickly used to further applications, such as analysis and recognition of shape [52]. Also, active contour method were extensively used for image segmentation due to find boundary of objects, fast and efficient method.

For active contour, there are two types involved edgebased, and region-based. For edge-based, an edge detector is often used to come up with the boundaries of image regions and to attract the contours to these boundaries. For regionbased, statistical information of the image intensities are used to evolve the contour [53]. Region-based active contours often have no restrictions on the placement of initial contours due to global energy minimization. That is, regardless of the position of initial contours, region-based active contour can detect internal borders. A method of autonomous segmentation is provided by the usage of predefined start contours. They are also less susceptible to local minima or noise than active contours based on edges. Thus, initializing active contour is a fundamental segmentation problem.

i. Snake: This model was proposed by Kass, Witkinand and Terzopoulos [54], which a spline is used. Because only local information along the contour is used, the classic snake can only provide an accurate placement of the edge if the beginning contour is given sufficiently near the edges. On the other hand, estimating the proper position of commencing contours without prior knowledge is a difficult process. Also, snakes keep the same topology throughout their evolution, therefore, unable to identify several boundaries at the same time. As a result, snakes are unable to merge from multiple initial contours or split to multiple boundaries. The level set methods provide a solution for solving the snake's problem.

ii. Active contour based regions: In the classical methods rely on the edge- function, which relies on the image gradient, to stop the curve evolution. However, the fundamental disadvantage of these methods is that they can only detect objects with gradient-defined borders, and in actuality, discrete gradients are bounded, and the stopping function is never zero near the edge, which could result in the curve leaking past the borders. In case of noisy images, isotropic smoothing Gaussian must be strong, smoothing the edges. This might lead to detection of wrong edges when the stopping function depends only on the gradient. The active contour model was proposed by Chan and Vese [55] as a solution to this problem. Curve evolution techniques are used. It was suggested that things whose borders are not defined by gradient be determined. To achieve the desired result, this model focuses on minimizing energy consumption. This method's major goal is to recognize objects even when there isn't a significant gradient.

2. Graph methods: It depends on graph theory based approaches. This class of segmentation methods is based on locating minimum cuts in a graph, with the cut criterion 
aiming to minimize the similarity between pixels being separated, , it contains:-

i. Normalize cut is a graph technique initially introduced by Shi and Malik [26]. This technique regards an image pixel as anode of graph and considers division as a chart apportioning issue. The image is demonstrated as a undirected, weighted diagram. Every pixel is a node in the graph, and an edge is framed between every pair of pixels. The heaviness of an edge is a measure of the comparability between the pixels. The image is divided into disjoint sets by evacuating the edges interfacing the portions. The ideal apportioning of the graph is the particular case that minimizes the weights of the edges that were evacuated [56].

ii. Graph - cut is only able to find a global optimum for binary labelling as foreground, background image segmentation. The cut should be made at the point where the object meets the background. Specifically, energy should be minimized near the object boundary [28]. It is optimized energy function over the segmentation.

iii. Local variation is a similar to normalized cuts. The dissimilarity between pixels is measured by the weights at each edge. This method divides an image into segments based on the degree of variability in adjacent regions [57, 58]. Another concern in image segmentation that has been tackled is the dealing with the aspect of under or over segmentation.

Graph methods have limitations. Firstly is high computational. Secondly it is sensitive to over and under segment. Thirdly need interactive with user and time complexity. An example of energy-based segment is presented in Fig. 5.

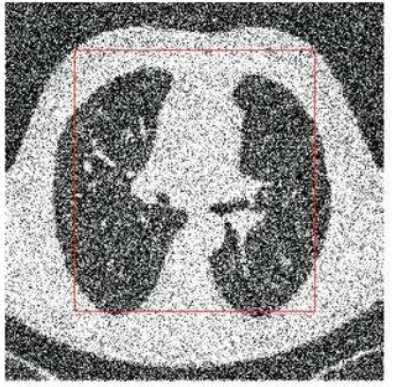

(a)

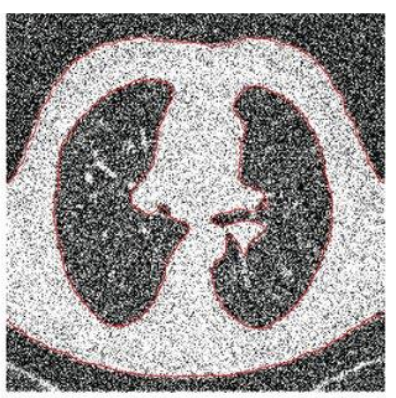

(b)
Fig. 5: Energy-based segment (a) original image, (b) the resulting image after segmentation [59]

The main four categories of image segmentation are depicted in the Table 2 with presenting the advantages and the disadvantages of each of them. Apparently, all the categories suffer from high computational complexity requirements to select some parameters correctly, and from some degree of complexity. Active contour is among the simplest approaches which enable integrating it with other computer vision or pattern recognition algorithms. Additionally, active contour can convergence to the shape of the segmented object accurately, which is important for the calories counting. Fig. 6 depicts the image segmentation techniques categories.

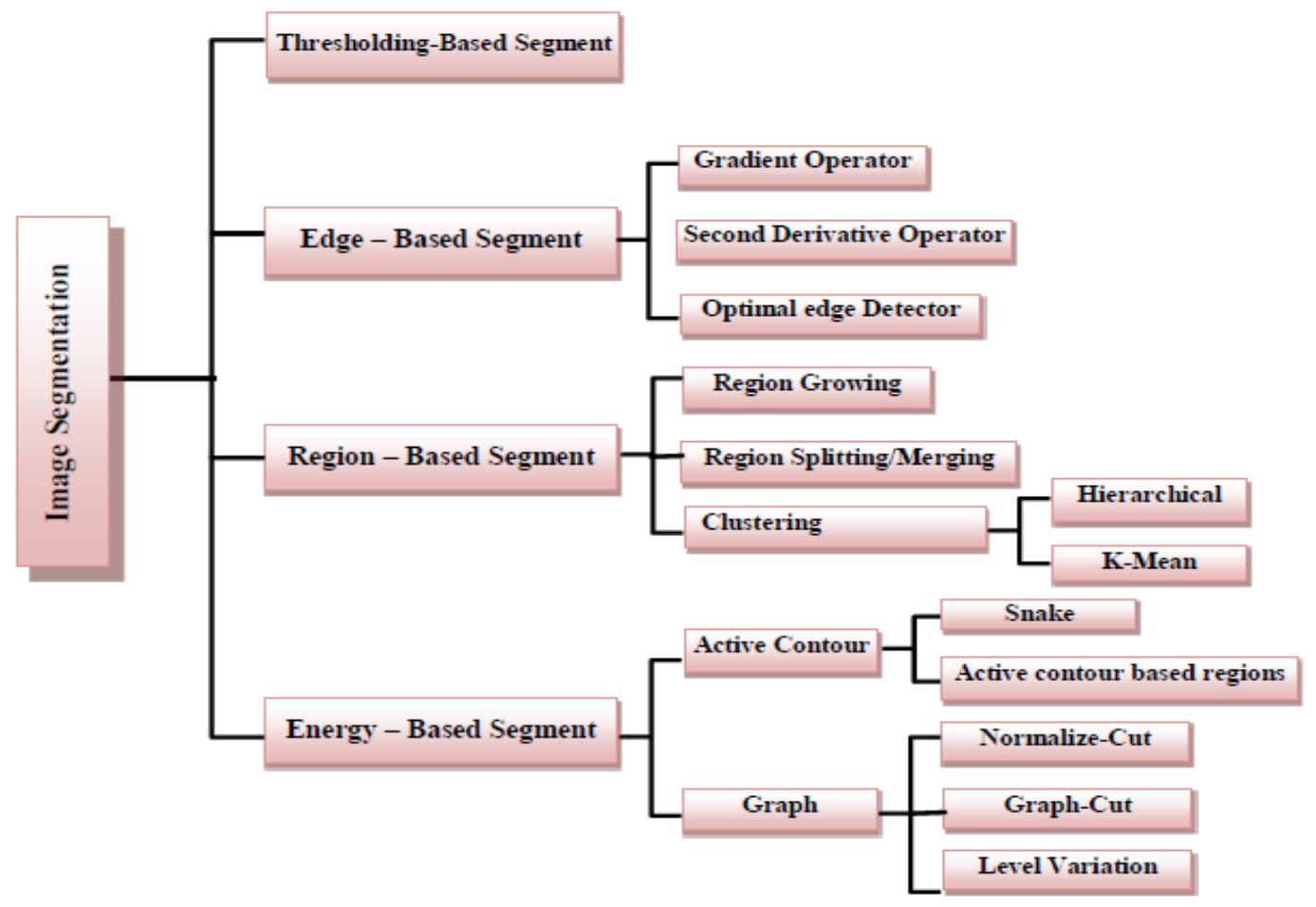

Fig. 6: Image segmentation techniques categories 
TABLE 2

SUMMARIES THE METHODS FOR IMAGE SEGMENTATION

\begin{tabular}{|c|c|c|c|}
\hline Category & Method & Advantages & Disadvantages \\
\hline 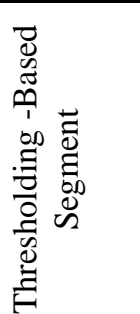 & Thresholding & $\begin{array}{l}\text { 1-It does not require any prior knowledge } \\
\text { of the image. } \\
\text { 2-It has a lower computational } \\
\text { difficulty.[18] } \\
\text { 3-It is benefit to isolate background and } \\
\text { foreground. }\end{array}$ & $\begin{array}{l}\text { 1- Significant peaks and dips in the image } \\
\text { are difficult to spot. } \\
\text { 2-It does not take into account the spatial } \\
\text { detail so the segmented regions may not be } \\
\text { contiguous. } \\
\text { 3- It is sensitive to noise. } \\
\text { 4-Hard to set threshold. [30] }\end{array}$ \\
\hline \multirow{3}{*}{ 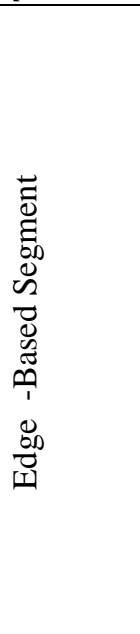 } & Gradient operator. & $\begin{array}{l}\text { 1-Easy, quick, and simple to compute. } \\
\text { 2-Edges and their orientation are } \\
\text { recognized.[32] }\end{array}$ & $\begin{array}{l}\text { 1- Noise sensitivity is greater in these. } \\
\text { 2-Edge detection can be shaky at times. } \\
\text { 3- Inconsistent.[21] }\end{array}$ \\
\hline & $\begin{array}{l}\text { Second derivative } \\
\text { operator. }\end{array}$ & $\begin{array}{l}\text { 1- Due to the approximation of gradient } \\
\text { magnitude, the cross operation detection of } \\
\text { edges and their orientation is } \\
\text { straightforward. } \\
\text { 2-In all directions, the properties are fixed. } \\
\text { 3-A wide area around the pixel can be } \\
\text { tested.[33] }\end{array}$ & $\begin{array}{l}\text { 1- As noise increases, the magnitude of } \\
\text { edges declines due to edge detection and } \\
\text { orientation. } \\
\text { 2- Faults in the gray level intensity function } \\
\text { in corners, curves, and where the gray level } \\
\text { intensity function fluctuates. }\end{array}$ \\
\hline & $\begin{array}{l}\text { Optimal edge } \\
\text { detector }\end{array}$ & $\begin{array}{l}\text { 1-The signal-to-noise ratio has been } \\
\text { improved. } \\
\text { 2-More accurate detection in noisy } \\
\text { environments.[34] }\end{array}$ & $\begin{array}{l}\text { 1- Complex computations that take a long } \\
\text { time to complete. } \\
\text { 2- Generic thresholds that operate on all } \\
\text { images are challenging to come up with.[34] }\end{array}$ \\
\hline \multirow{3}{*}{ 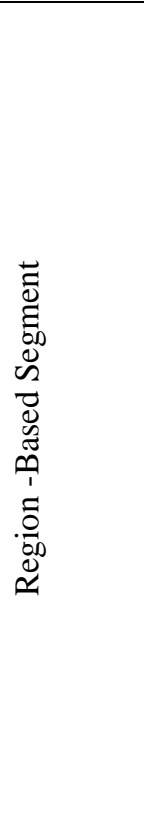 } & Region growing & $\begin{array}{l}\text { 1- Can correctly distinguish between } \\
\text { regions with the same attributes. } \\
\text { 2- Make sure the borders are clean. } \\
\text { 3-The principle is straightforward. } \\
\text { 4-Its results have a good shape matching. } \\
\text { 5-Can select many criteria at the same } \\
\text { time. [35] }\end{array}$ & $\begin{array}{l}\text { 1- Both in terms of processing time and } \\
\text { memory, this is a costly option. } \\
\text { 2- The seed region selected and the order in } \\
\text { which pixels and regions are evaluated are } \\
\text { intricately tied to region growth. } \\
\text { 3- Seed point can obtain by manual } \\
\text { interactive. } \\
\text { 4- Sensitive to noise and the region may be } \\
\text { disconnecting. [35] }\end{array}$ \\
\hline & $\begin{array}{l}\text { Region splitting and } \\
\text { merging }\end{array}$ & $\begin{array}{l}\text { 1- Split the image until get the desired } \\
\text { resolution. } \\
\text { 2-Different criteria might be used for the } \\
\text { splitting and merging criteria. [37] }\end{array}$ & $\begin{array}{l}\text { 1-Complex method and time-consuming } \\
\text { method. } \\
2 \text {-The outcome of region merging is usually } \\
\text { determined by the order in which regions are } \\
\text { merged. }\end{array}$ \\
\hline & Clustering & $\begin{array}{l}\text { 1-Fast speed, non-need of prior knowledge } \\
\text { about distribution of the data.Efficiency, } \\
\text { straightforwardness sand ability to cluster } \\
\text { huge data points very quickly. } \\
\text { 2-The concept is simple, because numbers } \\
\text { of cluster is fixed. [46] }\end{array}$ & $\begin{array}{l}\text { 1- Problem of cluster number selection. } \\
\text { 2- Various initial centroids will result in diff } \\
\text { erent outcomes. } \\
\text { 3- Sensitive to noise. [45] }\end{array}$ \\
\hline 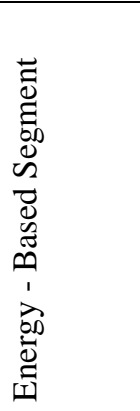 & Active contour & $\begin{array}{l}\text { 1-Find boundaries of objects. [54] } \\
\text { 2- It prefer to define images have a large } \\
\text { shape variety. } \\
\text { 3-Fast and efficient method. } \\
\text { 4- Provide a linear description of the object } \\
\text { shape during convergence without } \\
\text { requiring additional processing. } \\
\text { 5- It can immediately construct closed } \\
\text { parametric curves or surfaces from images } \\
\text { and apply a smoothness constraint on them. }\end{array}$ & $\begin{array}{l}\text { 1- Sensitive to noise. } \\
\text { 2- Sensitive to a choice of its parameters is } \\
\text { the number of initial contours and position. }\end{array}$ \\
\hline
\end{tabular}




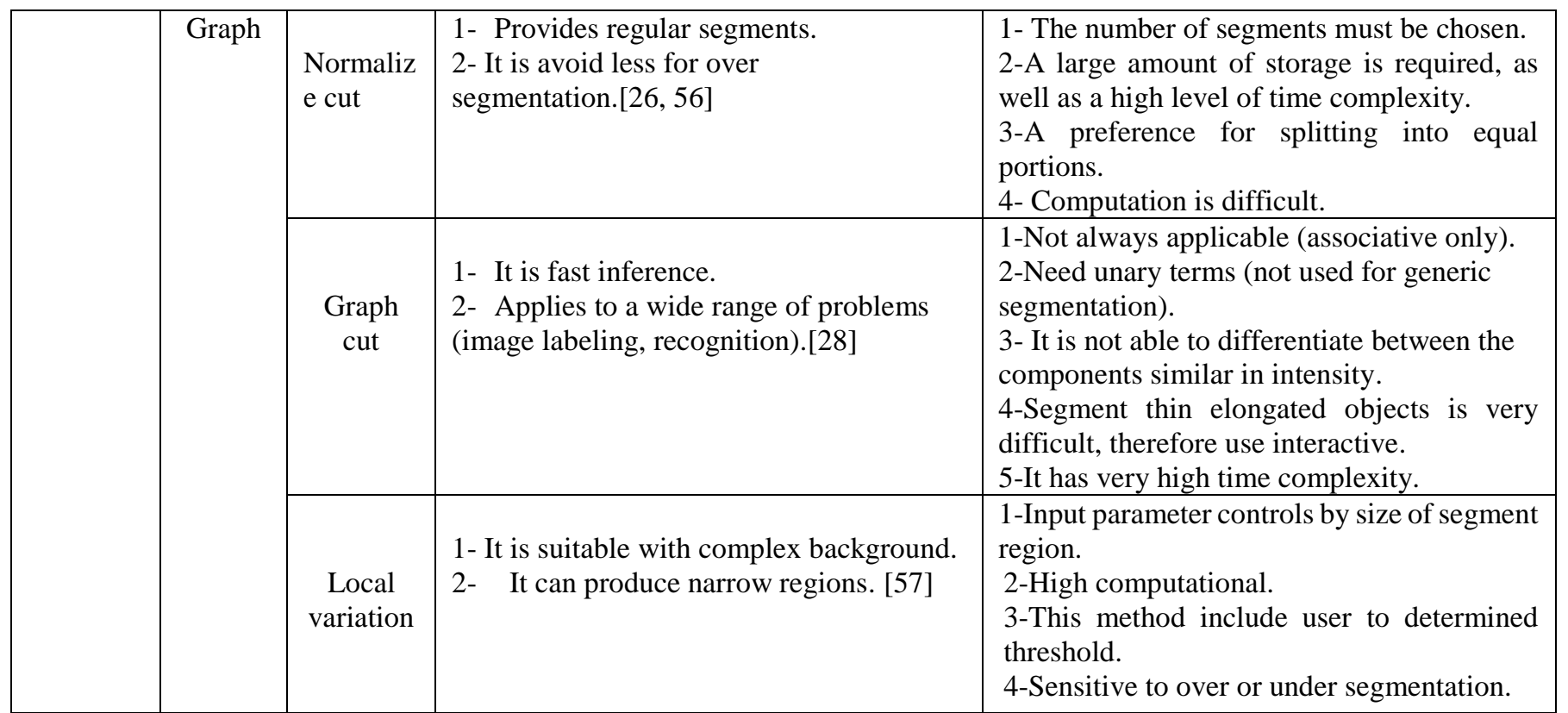

\section{IMAGE SEgMENTATION APPLICATIONS}

Image segmentation techniques have gained increasing and essential importance in a wide range of applications as computer technology has advanced [60]. Table 3 summarizes some real applications and some purposes of using segmentation techniques in such applications.

\section{RESULTS AND DISCUSSION}

The previous section provided a detailed review of the different segmentation methods. Through the review, it turns out how important segmentation is because it is widely used in real-world applications, such as pattern recognition, feature extraction, image retrieval, machine vision, satellite imaging, biometrics, and the military. The investigation of the pros and cons of segmentation methods with respect to the main segmentation criteria (i.e., noise sensitive, highly arithmetic, manual interaction, segmentation sensitive or under partition) revealed that it is difficult to achieve a satisfactory performance of the previous criteria at the same time. Therefore, image segmentation should not be ignored, and it is preferable to combine segmentation techniques to reduce its disadvantages in order to obtain better results.

\section{CONCLUSION AND FUTURE WORK}

This study provides a review of different segmentation techniques due to their importance in digital image processing such as border, region, and hybrid techniques in real-world applications. Despite several decades of research, image segmentation is still a difficult challenge in image processing, as each method has advantages and disadvantages. So there is no single technology that can be considered good for all types of images and not all technologies are equally suitable for a particular type of image. Future work is a review of the use of intelligent technologies such as deep learning and optimization algorithms in image segmentation.

\section{CONFLICT OF INTEREST}

The authors have no conflict of relevant interest to this article.

\section{REFERENCES}

[1] B. Desai, U. Kushwaha, S. Jha, \& M. S. NMIMS, "Image filtering-Techniques Algorithms and Applications," Applied GIS, vol. 7, no. 11, pp. 970-975, 2020.

[2] N. Dey, \& A. S. Ashour, " Meta-heuristic algorithms in medical image segmentation: a review," Advancements in Applied Metaheuristic Computing, pp.185-203. 2018.

[3] P. Shashi, \& R. Suchithra, " Review Study on Digital Image Processing and Segmentation," Am. J. Comput. Sci. Technol, vol.2, no.68, 2019.

[4] S. K. Abdulateef, S. R. A. AHMED, \& M. D. Salman, "A Novel Food Image Segmentation Based on Homogeneity Test of K-Means Clustering," In IOP Conference Series: Materials Science and Engineering, vol.928, no.3, p. 032059, 2020.

[5] A. S. Al-Waisy, R. Qahwaji, S. Ipson, S. Al-Fahdawi, \&T. A. Nagem, "A multi-biometric iris recognition system based on a deep learning approach," Pattern Analysis and Applications, vol.2, no.3, pp.783-802, 2018.

[6] A. Garcia-Garcia, S. Orts-Escolano, S. Oprea, V. VillenaMartinez,P.Martinez-Gonzalez,\&J. Garcia-Rodriguez, "A survey on deep learning techniques for image and video semantic segmentation," Applied Soft Computing, vol.70, pp.41-65, 2018.

[7] A. Pfeuffer, K. Schulz, \& K. Dietmayer, "Semantic segmentation of video sequences with convolutional lstms," In IEEE Intelligent Vehicles Symposium (IV), pp. 1441-1447, 2019.

[8] P. Thakur, \& N. Madaan,"A survey of image segmentation techniques," International Journal of Research in Computer Applications and Robotics, vol.2, no.4, pp.158-165, 2014. 
TABLE 3.

SUMMARIES OF SOME APPLICATIONS

\begin{tabular}{|c|c|c|}
\hline Seq. & Real-world Applications & Purpose \\
\hline $1-$ & Medical applications & $\begin{array}{l}\text { 1- To detect MRI using region growing [35]. } \\
\text { 2- Measuring Tissue volumes } \\
\text { Image-guided therapy is one of the most important methods for accurate and rapid } \\
\text { diagnosis of diseases, as image segmentation is useful that allows for easy and } \\
\text { accurate analysis }[61,2] \text {. }\end{array}$ \\
\hline $2-$ & Pattern Recognition & 1- Face [62] and Iris [63]. \\
\hline $3-$ & Remote sensing & $\begin{array}{l}\text { 1- To segment satellite images. } \\
\text { The segment satellite images for instance in urban planning or precision agriculture } \\
\text { to treatment major environmental issues that may affect a changing climate [55] }\end{array}$ \\
\hline 4- & Earth observation (EO) & $\begin{array}{l}\text { 1- Road network. } \\
\text { 2- Local climate zones. } \\
\text { 3- Traffic control systems. } \\
\text { The dynamic analysis of Earth's surface through traditional approaches gives an } \\
\text { increase in data, to extract high-resolution data that helps to better understand the } \\
\text { dynamics of the Earth's surface have used several methods, including a deep learning } \\
\text { model or convolutional neural networks (CNNs) [64]. }\end{array}$ \\
\hline $5-$ & Calories estimation & $\begin{array}{l}\text { Calorie estimation utilizing a mobile phone to separate the image's background from } \\
\text { the items that make it up [65]. }\end{array}$ \\
\hline 6- & Shape Detection & $\begin{array}{l}\text { It is an important part of image processing to detecting boundary from image parts } \\
\text { which differ in color, brightness, or texture [66]. }\end{array}$ \\
\hline $7-$ & Security & $\begin{array}{l}\text { 1- Object recognition and matching. } \\
\text { 2- Scene understanding. } \\
\text { 3- Fingerprint [67]. } \\
\text { 4- Video surveillance } \\
\text { Security is a critical and difficult issue. Image segmentation is used as a major pre- } \\
\text { processing in many applications such as security monitoring by separating a given } \\
\text { image into many salient parts [68]. }\end{array}$ \\
\hline $8-$ & $\begin{array}{l}\text { Industrial Automation } \\
\text { applications [55] }\end{array}$ & $\begin{array}{l}\text { 1- Drones } \\
\text { 2- Self- driving cars } \\
\text { 3- Robotics }\end{array}$ \\
\hline $9-$ & Image Processing [69] & $\begin{array}{l}\text { 1- image-sharpening. } \\
\text { 2- Color and video processing }\end{array}$ \\
\hline $10-$ & Agricultural imaging & - Detection of crops' disease [70]. \\
\hline
\end{tabular}

[9] I. R. I. Haque, \& J. Neubert, "Deep learning approaches to biomedical image segmentation," Informatics in Medicine Unlocked, vol.18, 100297, 2020.

[10] R. E. Armya, \& A. M. Abdulazeez, " Medical images segmentation based on unsupervised algorithms: A review," Qubahan Academic Journal, vol. 1, no.2, pp.7180, 2021.

[11] S. K. Abdulateef, M. Mahmuddin, \& N. H. Harun, "Developing a new features approach for colour food image segmentation," ARPN Journal of Engineering and Applied Sciences, vol.12, no. 23, pp. 6904-6910, 2017.

[12] S. K. Abdulateef, M. Mahmuddin, N. H. Harun, \& Y. Aljeroudi, "Dietary assessment and obesity aviodance system based on vision: A review," Proceedings of the 5th International Conference on Computing and Informatics, ICOCI 2015, Turkey. pp. 651-658, 2015.

[13] N. M. Zaitoun, \& M. J. Aqel, "Survey on image segmentation techniques". Procedia Computer Science, vol.65, pp.797-806, 2015.

[14] W. Min, S. Jiang, L. Liu, Y. Rui, \& R. Jain, "A survey on food computing," ACM Computing Surveys (CSUR), vol. 52, no.5, pp. 1-36, 2019.

[15] I. Chen, H. Shen, \& F. Wang, "Quantifying the thickness of each color material in multilayer transparent specimen based on transmission image," Textile Research Journal, vol. 90, no.21-22, pp.2522-2532, 2020. 
[16] P. Pouladzadeh, S. Shirmohammadi, \& A. Yassine, "Using graph cut segmentation for food calorie measurement," In Proceedings of MeMeA: The IEEE International Symposium on Medical Measurements and Applications, pp.1-6,2014.

[17] C. J. Boushey, M. Spoden, F. M. Zhu, E. J. Delp, \& D. A. Kerr, "New mobile methods for dietary assessment: review of image-assisted and image-based dietary assessment methods," Proceedings of the Nutrition Society, vol. 76, no. 3, pp. 283-294, 2017.

[18] C. Tulasigeri, \& M. Irulappan, "An advanced thresholding algorithm for diagnosis of glaucoma in fundus images," In 2016 IEEE International Conference on Recent Trends in Electronics, Information \& Communication Technology (RTEICT), pp. 1676-1680, 2016.

[19] A. Garg, "A Review on Image Segmentation Techniques," International Journal of Recent Research Aspects (IJRRA), pp. 53-55, 2016.

[20] R. Nirgude, \& S. Jain, "Color image segmentation with k- means clustering and dynamic region merging," Journal of Science, Engineering \& Technology: A Peer Reviewed National Journal, vol.1, no.5, pp.1-10, 2014.

[21] B. K. Shah, V. Kedia, R. Raut, S. Ansari, \& A. Shroff, "Evaluation and Comparative Study of Edge Detection Techniques," IOSR Journal of Computer Engineering, vol. 22, no. 5, pp. 6-15, 2020.

[22] S. Fan, Y. Sun, \& P. Shui, "Region-merging method with texture pattern attention for SAR image segmentation," IEEE Geoscience and Remote Sensing Letters,vol.18, no.1, pp.112-116. 2020.

[23] J. Bhattacharjee, S. Santra, \& A. Deyasi, "A Metaheuristic Approach for Image Segmentation Using Genetic Algorithm," In Advances in Smart Communication Technology and Information Processing: OPTRONIX, pp. 125-134, 2021.

[24] D. Kumar, R. K. Agrawal, \& P. Kumar, "Bias-corrected intuitionistic fuzzy c-means with spatial neighborhood information approach for human brain MRI image segmentation," IEEE Transactions on Fuzzy Systems, 2020.

[25] P. Pouladzadeh, S. Shirmohammadi, \& T. Arici, "Intelligent SVM based food intake measurement system," In Proceedings of CIVEMSA: The IEEE International Conference on Computational Intelligence and Virtual Environments for Measurement Systems and Applications, pp. 87-92, 2013.

[26] L. C. Chen, G. Papandreou, I. Kokkinos, K. Murphy, \& A. L. Yuille, "Deeplab: Semantic image segmentation with deep convolutional nets, atrous convolution, and fully connected crfs," IEEE transactions on pattern analysis and machine intelligence, vol. 40, no. 4, pp. 834-848, 2017.

[27] K. Ding, L. Xiao, \& G. Weng, "Active contours driven by local pre-fitting energy for fast image segmentation," Pattern Recognition Letters, vol.104, pp.29-36, 2018.

[28] W. Wang, Z. Li, J. Yue, \& D. Li, "Image segmentation incorporating double-mask via graph cuts," Computers \& Electrical Engineering, vol. 54, pp. 246-254, 2016.
[29] X. Zheng, Q. Lei, R. Yao, Y. Gong, \& Q. Yin. "Image segmentation based on adaptive K-means algorithm," EURASIP Journal on Image and Video Processing, vol.2018, no. 1, pp.1-10, 2018.

[30] F. M. Abubakar, "Study of image segmentation using thresholding technique on a noisy image," International Journal of Science and Research (IJSR), vol.2, no.1, pp.49-51, 2013.

[31] Thresholding(imageprocessing). http://en.wikipedia.org/w/index.php?title=Thresholding (image_processing)\&oldid=606970852, 2014. [Online; Accessed August -2014].

[32] S. Chakraborty, "An advanced approach to detect edges of digital images for image segmentation," In Applications of Advanced Machine Intelligence in Computer Vision and Object Recognition: Emerging Research and Opportunities, pp. 90-118, 2020.

[33] S. E. R. T. Eser, \& A. V. C. I. Derya, "A new edge detection approach via neutrosophy based on maximum norm entropy," Expert Systems with Applications, vol.115, pp.499-511, 2019.

[34] D. Sangeetha, \& P. Deepa, " FPGA implementation of cost-effective robust Canny edge detection algorithm," Journal of Real-Time Image Processing, vol.16, no.4, pp.957-970, 2019.

[35] N. S. M. Raja, S. L. Fernandes, N. Dey, S. C. Satapathy, \& V. Rajinikanth, "Contrast enhanced medical MRI evaluation using Tsallis entropy and region growing segmentation," Journal of Ambient Intelligence and Humanized Computing, 1-12, 2018.

[36] A. Khwairakpam, R. A. Hazarika, \& D. Kandar, "Image segmentation by fuzzy edge detection and region growing technique," In Proceedings of the Third International Conference on Microelectronics, Computing and Communication Systems, pp. 51-64, 2019.

[37] J. Acharya, S. Gadhiya, \& K. Raviya, "Segmentation techniques for image analysis: A review," International Journal of Computer Science and Management Research, vol.2, no.1, pp.1218-1221, 2013.

[38] T. Wang, L. Yin, \& X. Wang, "A community detection method based on local similarity and degree clustering information," Physica A: Statistical Mechanics and its Applications, vol.490, pp.1344-1354, 2018.

[39] K. Shrivastava, N. Gupta, \& N. Sharma, "Medical image segmentation using modified $\mathrm{k}$ means clustering," International Journal of Computer Applications, vol.103, no.16, pp.12-16, 2014.

[40] M. Satokangas, S. Lumme, M. Arffman, \& I. Keskimäki,"Trajectory modelling of ambulatory care sensitive conditions in Finland in 1996-2013: assessing the development of equity in primary health care through clustering of geographic areas-an observational retrospective study," BMC health services research, vol.19, no.1, pp.1-12, 2019.

[41] D. P. Mashinini, K. J. Fogarty, R. C. Potter, \& J. D. Berles, "Geographic hot spot analysis of vaccine exemption clustering patterns in Michigan from 2008 to 2017," Vaccine, vol.38, no.51, pp.8116-8120, 2020.

[42] A. Aher, J. Kasar, P. Ahuja, \& V. Jadhav, "Smart agriculture using clustering and IOT". International 
Research Journal of Engineering and Technology (IRJET), vol.5, no. 3, pp.2395-0056, 2018.

[43] H. Xiao, L. He, X. Li, Q. Zhang, \& W. Li, " Texture synthesis: a novel method for generating digital models with heterogeneous diversity of rock materials and its CGM verification," Computers and Geotechnics, vol.130, 103895, 2021.

[44] V. Cohen-Addad, V. Kanade, F. Mallmann-Trenn, \& C. Mathieu, "Hierarchical clustering: Objective functions and algorithms," Journal of the ACM (JACM), vol. 66, no.4, pp.1-42, 2019.

[45] Q. Xu, Q. Zhang, J. Liu, \& B. Luo, "Efficient synthetical clustering validity indexes for hierarchical clustering," Expert Systems with Applications, vol.151, 113367, 2020.

[46] J. Xu, \& K. Lange, "Power k-means clustering," In International Conference on Machine Learning, pp. 69216931, 2019.

[47] T. Ege, Y. Ando, R. Tanno, W. Shimoda, \&K. Yanai, "Image-based estimation of real food size for accurate food calorie estimation," In 2019 IEEE Conference on Multimedia Information Processing and Retrieval (MIPR) pp. 274-279, 2019.

[48] S. Turmchokkasam, \& K. Chamnongthai, "The design and implementation of an ingredient-based food calorie estimation system using nutrition knowledge and fusion of brightness and heat information," IEEE Access,vol. 6, pp.46863-46876, 2018.

[49] C. Zhu, C. U. Idemudia, \& W. Feng, "Improved logistic regression model for diabetes prediction by integrating PCA and K-means techniques," Informatics in Medicine Unlocked, vol. 17, 100179, 2019.

[50] B. R. Lee, "An image segmentation approach for fruit defect detection using k-means clustering and graph-based algorithm," Vietnam Journal of Computer Science, vol. 2, no. 1, pp. 25-33, 2015.

[51] A. González-López, J. de Moura, J. Novo, M. Ortega, \&M. G. Penedo, "Robust segmentation of retinal layers in optical coherence tomography images based on a multistage active contour model," Heliyon, vol. 5, no. 2, e01271, 2019.

[52] L. Yang, D. Xin, L. Zhai, F. Yuan, \&X. Li, " Active contours driven by visual saliency fitting energy for image segmentation in SAR images," In 2019 IEEE 4th International Conference on Cloud Computing and Big Data Analysis (ICCCBDA) pp. 393-397, 2019.

[53] H. Yu, F. He, \& Y. Pan, "A survey of level set method for image segmentation with intensity inhomogeneity," Multimedia Tools and Applications, vol.79, no.39, pp. 28525-28549, 2020.

[54] Z. Zhang, C.Duan, T.Lin, S. Zhou, Y. Wang, \& X. Gao, " GVFOM: a novel external force for active contour based image segmentation," Information Sciences, vol.506, pp.1$18,2020$.

[55] S. Minaee, Y. Y. Boykov, F. Porikli, A. J.Plaza, N. Kehtarnavaz, \& D. Terzopoulos, "Image segmentation using deep learning: A survey," IEEE Transactions on Pattern Analysis and Machine Intelligence, 2021.

[56] G. Dimauro, \& L. Simone, "Novel biased normalized cuts approach for the automatic segmentation of the conjunctiva," Electronics, vol.9, no.6, 997, 2020.
[57] P. F. Felzenszwalb, \& D. P. Huttenlocher, "Efficient graph-based image segmentation," International Journal of Computer Vision, vol.59, no.2, pp.167-181, 2014.

[58] P. Subudhi, \& S. Mukhopadhyay, "A statistical active contour model for interactive clutter image segmentation using graph cut optimization," Signal Processing, vol.184, 108056, 2021.

[59] S. Zafari, T. Eerola, J. Sampo, H. Kälviäinen, \& H. Haario, " Segmentation of partially overlapping convex objects using branch and bound algorithm," In Asian Conference on Computer Vision, pp. 76-90. 2016.

[60] K. Jeevitha, A. Iyswariya, V. RamKumar, S. M. Basha, \& V. P. Kumar, "A review on various segmentation techniques in image processing," European Journal of Molecular \& Clinical Medicine, vol.7, no.4, pp.13421348, 2020.

[61] A. Abdulrahman, \& S. Varol, "A Review of Image Segmentation Using MATLAB Environment," In 2020 8th International Symposium on Digital Forensics and Security (ISDFS) pp. 1-5, 2020.

[62] Y. Kortli, M. Jridi, A. Al Falou, \& M. Atri,"Face recognition systems: A Survey," Sensors, vol.20, no.2, pp.1-36, 2020.

[63] M. Trokielewicz, A. Czajka, \& P. Maciejewicz, "Postmortem iris recognition with deep-learning-based image segmentation," Image and Vision Computing, vol.94, 103866, 2020.

[64] T. Hoeser, F. Bachofer, \& C. Kuenzer, "Object detection and image segmentation with deep learning on Earth observation data: A review-Part II: Applications," Remote Sensing, vo. 12, no. 18, 3053, 2020.

[65] T. Ege, Y. Ando, R. Tanno, W. Shimoda, \& K. Yanai, "Image-based estimation of real food size for accurate food calorie estimation," In 2019 IEEE Conference on Multimedia Information Processing and Retrieval (MIPR), pp. 274-279, 2019.

[66] Y. Yuan, J. Xie, X. Chen, \& J. Wang, "Segfix: Modelagnostic boundary refinement for segmentation," In European Conference on Computer Vision, pp. 489-506, 2020.

[67] E. M. Cherrat, R. Alaoui, \& H. Bouzahir, "Improving of fingerprint segmentation images based on K-means and DBSCAN clustering," International Journal of Electrical \& Computer Engineering (2088-8708), vol.9, no.4, pp. 2425-2432, 2019.

[68] J. Ren, G. Chen, X. Li, \& K. Mao, "Striped-texture image segmentation with application to multimedia security," Multimedia Tools and Applications, vol.78, no.19, pp.26965-26978, 2019.

[69] A. P. Agrawal, \& N. Tyagi, "Review on digital image segmentation techniques," Journal of Critical Reviews, vol.7, no. 3, pp. 779-784, 2020.

[70] S. Kalaivani, S. P. Shantharajah, \& T. Padma, "Agricultural leaf blight disease segmentation using indices based histogram intensity segmentation approach," Multimedia Tools and Applications, vol. 79, no.13,pp.9145-9159,2020. 